\title{
Hydrodynamic analysis of oscillating water column wave energy devices
}

\author{
Harry B. Bingham ${ }^{1} \cdot$ Damien Ducasse $^{3} \cdot$ Kim Nielsen $^{2} \cdot$ Robert Read $^{1}$
}

Received: 20 January 2015 / Accepted: 15 June 2015 / Published online: 3 July 2015

(C) Springer International Publishing AG 2015

\begin{abstract}
A 40-chamber I-Beam attenuator-type, oscillating water column, wave energy converter is analyzed numerically based on linearized potential flow theory, and experimentally via model test experiments. The high-order panel method WAMIT by Newman and Lee (WAMIT; a radiation-diffraction panel program for wave-body interactions, 2014, http://www.wamit.com) is used for the basic wave-structure interaction analysis. The damping applied to each chamber by the power take off is modeled in the experiment by forcing the air through a hole with an area of about $1 \%$ of the chamber water surface area. In the numerical model, this damping is modeled by an equivalent linearized damping coefficient which extracts the same amount of energy over one cycle as the experimentally measured quadratic damping coefficient. The pressure in each chamber in regular waves of three different height-tolength ratios is measured in the experiments and compared to calculations. The model is considered in both fixed and freely floating, slack-moored conditions. Comparisons are also made to experimental measurements on a single fixed
\end{abstract}

Harry B. Bingham

hbb@mek.dtu.dk

Damien Ducasse

damienducasse@seureca.com

Kim Nielsen

KIN@ramboll.com

Robert Read

rrea@mek.dtu.dk

1 Department of Mechanical Engineering, Technical University of Denmark, 2800 Lyngby, Denmark

2 Rambøll A/S, Hannemanns Allé 53, 2300 Copenhagen, Denmark

3 SEURECA Muscat LLC, Jibroo, Al Azaiba, Muscat, Sultanate of Oman chamber. The capture width ratio in each case is predicted based on the pressures in the chambers. Good agreement is found between the calculations and the experiments.

Keywords Wave energy conversion - Oscillating water columns - Marine hydrodynamics · Numerical methods . Model testing

\section{Introduction}

Motivated by a desire to decrease global emissions of carbon and other environmental pollutants, the world is moving steadily to replace fossil fuel-based energy supplies with renewable sources. Ocean wave energy has the potential to make a significant contribution to this effort. A detailed description of the resource is given in Barstow et al. (2008). Estimates for the realistically exploitable resource vary, but according to the above cited work it is on the order of 10-25\% of current electricity consumption, while a recent comprehensive study by the Electric Power Research Institute in Jacobsen (2011) estimates that in the USA it could be as much as $1 / 3$ of current demand. Some countries have even larger potentials. Wave energy converter (WEC) technology is still relatively young compared to wind, water, solar and even tidal power extraction, and it thus faces significant hurdles to achieving economically competitive designs. Significant political activity has recently been initiated, however, to mature the industry and speed the large-scale deployment of wave energy installations, see for example: IEA (2014), OEE (2014) and SIOcean (2012).

The large-scale exploitation of wave energy will most likely progress gradually from breakwater-based and nearshore devices (e.g. WAVENERGY.IT 2014; Oyster 2014) to smaller- and finally larger-scale deep water sites. The 
progression to deeper water will ideally take place together with wind power to exploit the combined use of infrastructure, maintenance services and the shielding effect of the WECs on the wind turbines. Compared to point absorber and terminator WECs, in deep water attenuator devices have high theoretical absorption width and low mooring loads. The low loads are due to the exploitation of internal (non-rigid-body mode) degrees of freedom and a subsequent cancelation of forces along the long ship-like structure oriented parallel to the main direction of wave propagation. The degrees of freedom which are used to extract energy from the waves can be hinged motions (e.g. Pelamis 2014), individual mechanical oscillators (e.g. Wavestar 2014), or Oscillating Water Column (OWC) chambers. OWC chambers have the additional attractive feature of having no moving parts in the water, albeit at the expense of a generally reduced Power Take Off (PTO) conversion efficiency associated with existing air turbines compared to hydraulic systems. OWC-type modes also offer less freedom in tuning for resonant motions compared to mechanical oscillators. In-depth presentations of the history and analysis of WECs are given by for example: Falnes (2002), Cruz (2008), Drew et al. (2009), Falcão (2010), López et al. (2013) and McCormick (2007).

The OWC is one of the oldest and most highly developed forms of wave energy conversion, having been used in the late 1800 s to produce whistling buoys to warn ships about dangerous waters, and since the 1940s as electrical generators for navigational buoys. Several shore-based power generation installations have operated successfully for many years and a number of floating concepts have been tested over the years. Heath (2012) and CarbonTrust (2005) provide good overviews of the historical development of the OWC. Research and development activity in this area has accelerated dramatically in recent years. A practical example is the new U-type OWC design recently developed by Boccotti $(2007,2012,2015)$ and Boccotti et al. (2007). This design shows dramatically improved absorption width for fixed installations and is currently being installed in breakwaters at several locations.

The goal of this paper is to clarify a number of subtle details associated with the use of standard radiationdiffraction theory for the analysis of wave energy devices which include OWC chambers for wave energy extraction. By standard radiation-diffraction theory, we mean linearized potential flow theory solved in the frequency domain, and we employ here the WAMIT software of Newman and Lee (2014). Thus all viscous and non-linear effects are neglected, although we illustrate how iteration can be used to approximate the nonlinear behavior of the air turbine on the OWC chamber response. We also demonstrate that the calculations compare well with experimental measurements for two test cases with one and twenty six degrees of freedom, respectively. The concept chosen for analysis is a variant of the I-Beam Attenuator of Moody (1980) which we call the KNSwing. The original inspiration for this concept comes from the Kaimei project, see Masuda (1979) and Ishii et al. (1982), which ran from 1974 through the mid 1980s in Japan and was designed by Yoshio Masuda who also invented the above mentioned OWC-powered navigational buoys. The model considered here consists of forty chambers, each with internal dimensions of $6 \mathrm{~m}$ by $5 \mathrm{~m}$ by $7.5 \mathrm{~m}$, giving a total length of $150 \mathrm{~m}$. The target deployment area is the Danish North Sea, so the chambers are tuned to resonate at wave periods of around $6 \mathrm{~s}$. Model test experiments at a scale of 1:50 are carried out on the full model in both fixed and slackmoored conditions, and on on a single, fixed, double-chamber section. The PTO turbine is modeled by a hole in the chamber lid of approximately $1 \%$ of the chamber surface area which is an approximate model for an impulse turbine. Calculations in the frequency domain are made by estimating an equivalent linearized effective PTO damping coefficient. The calculations generally agree well with the measured data. A weakly non-linear time domain model is currently under development, which we expect to give even better performance.

\section{Theory}

Following the work of Evans (1982), standard first-order, radiation-diffraction theory can be extended to include the response of one or more partially enclosed OWC chambers, together with the usual rigid-body motions. The theory is also discussed in Falnes (2002), Lee and Nielsen (1996) and Lee et al. (1996) where the last two references have a particular focus on the implementation in WAMIT. We review the theory here in some detail for completeness and to highlight several subtle details which are not entirely obvious in the above mentioned references.

The flow is assumed to be described by a total velocity potential $\Phi(\mathbf{x}, t)$, satisfying the Laplace equation $\nabla^{2} \Phi=0$ in the fluid, along with linearized boundary conditions on the mean free surface at $z=0$ and on the mean wetted-body surface $S_{0}$, along with a no-flux condition on the solid sea bottom. As the problem is linear, $\Phi$ can be represented by the superposition of a number of time harmonic solutions at different frequencies $\omega$ such that

$\Phi(\mathbf{x}, t)=\Re\left\{\phi(\mathbf{x}, \omega) \mathrm{e}^{\mathrm{i} \omega t}\right\}$

where $\mathfrak{R}\{\}$ indicates the real part of a complex quantity. Having assumed linearity, we will also require the PTO to be a linear function of the response. In the context of existing frequency domain codes, it is convenient to treat the wave interaction with the internal pressure surface by defining an extended set of radiation modes $j=7,8, \ldots, 6+M_{p}$; each 


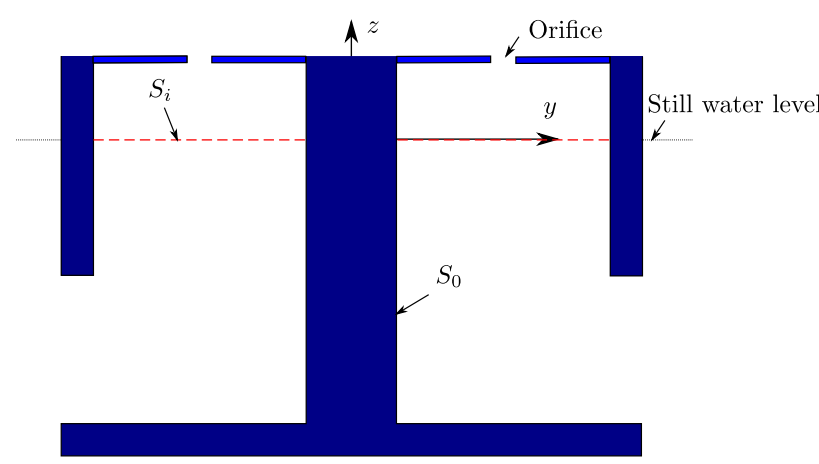

Fig. 1 A schematic horizontal cross-section of an OWC chamber section

of which has a predefined mode shape, with $M_{p}$ the total number of OWC modes defined. Each pressure mode is assigned a complex amplitude $\xi_{j}$ and a mode shape, $n_{j}(x, y)$ such that the total pressure applied to the interior free surface of the chamber $S_{i}$ is given by

$p_{0}(x, y)=-\rho g \sum_{j=7}^{6+M_{p}} \xi_{j} n_{j}(x, y)$.

The body surface is now extended to include the internal chamber free surface by defining $S_{b}=S_{0}+S_{i}$, and the generalized unit normal vector is set to zero on $S_{i}$ for the rigid-body modes $j=1,2, \ldots, 6$; and set to zero on $S_{0}$ for the pressure modes $j>6$. Figure 1 illustrates the surfaces for a horizontal cross-section of a single symmetric, doublechamber OWC section. The applied pressure modifies the Bernoulli equation on the free surface to give

$$
\left.\begin{array}{rl}
\mathrm{i} \omega \phi+g \zeta & =-\frac{p_{0}}{\rho} \\
\mathrm{i} \omega \zeta-\partial_{z} \phi & =0
\end{array}\right\} \quad \text { on } \quad z=0
$$

which can be combined with (2) to write

$\partial_{z} \phi-\frac{\omega^{2}}{g} \phi=-\frac{\mathrm{i} \omega}{\rho g} p_{0}=\mathrm{i} \omega \sum_{j=7}^{6+M_{p}} \xi_{j} n_{j}, \quad$ on $\quad z=0$

where $\partial_{z}$ indicates partial differentiation with respect to the $z$-coordinate. The potential is now decomposed into radiation and diffraction components in the usual way

$\phi=\phi_{R}+\phi_{D}, \quad \phi_{D}=A\left(\phi_{I}+\phi_{S}\right)$,

$\phi_{R}=\mathrm{i} \omega \sum_{j=1}^{6+M_{p}} \xi_{j} \phi_{j}$

where $A$ is the incident wave amplitude.

The diffraction potential $\phi_{D}$ represents the solution for a fixed body, with $p_{0}=0$, subjected to a wave of amplitude
$A$ incident from heading angle $\beta$ measured from the positive $x$-axis. This incident wave is defined by

$\phi_{I}=\frac{\mathrm{i} g}{\omega} \frac{\cosh [k(z+h)]}{\cosh (k h)} \mathrm{e}^{-\mathrm{i} k(x \cos \beta+y \sin \beta)}$

where the wave number $k=2 \pi / \lambda$ is related to the wave frequency via the dispersion relation

$\omega^{2}=g k \tanh (k h)$

with $h$ the water depth, $g$ the gravitational acceleration and $\lambda$ the wave length. The wave phase and group velocities are given by

$c=\frac{\omega}{k}, c_{g}=\frac{c}{2}\left(1+\frac{2 k h}{\sinh (2 k h)}\right)$.

The forcing in the diffraction problem comes only from the solid body boundary condition

$\partial_{n} \phi_{S}=-\partial_{n} \phi_{I} \quad$ on $\quad S_{0}$

where $\partial_{n}=\mathbf{n} \cdot \nabla$ indicates the derivative in the direction normal to the surface. The canonical radiation potentials $\phi_{j}$ correspond either to unit amplitude rigid-body motion or applied oscillatory pressure on the interior free surface, at frequency $\omega$. They are combined via the generalized boundary conditions

$$
\begin{aligned}
\partial_{z} \phi_{j}-\frac{\omega^{2}}{g} \phi_{j} & =n_{j}, \quad \text { on } \quad S_{i} \\
\partial_{n} \phi_{j} & =n_{j}, \quad \text { on } \quad S_{0}
\end{aligned}
$$

where the rigid-body modes and the pressure-surface modes are automatically distinguished by the extended definition of the normal vector $n_{j}$. In this way, the pressure modes are expressed in the same basic form as the normal rigid-body modes.

In an OWC, power is extracted from the waves using the motion of the water in the chamber to force air through a turbine. Thus, the critical quantity here is the flux of air through the chamber. The set of mode shapes $n_{j}$ which define the pressure distribution inside the chamber can be chosen as desired, but the most obvious choice is a set of orthogonal standing modes inside the chamber. With this choice, the mean (piston-mode) elevation inside a chamber will be the only mode that contributes to the flux through the turbine. In this work, we will consider only one mode for each chamber with $n_{j}=(0,0,1)$ on the internal free surface of chamber $j$ and zero everywhere else on $S_{b}$. We note, however, that the higher modes can influence the flux indirectly since all the radiation and pressure modes are coupled via the equations 
of motions. If large standing mode oscillations are expected to occur, then these modes should be included in the analysis.

The total volume flux $q$ through the chamber is given by

$$
\begin{aligned}
q & =\int_{S_{i}} \partial_{z} \phi \mathrm{d} S=\int_{S_{i}} \partial_{z} \phi_{D} \mathrm{~d} S+\int_{S_{i}} \partial_{z} \phi_{R} \mathrm{~d} S \\
& =q_{D}+q_{R} .
\end{aligned}
$$

The mean power extracted by the turbine over one period of oscillation is given by

$W=\frac{1}{T} \int_{0}^{T} \Re\left\{p_{0} \mathrm{e}^{\mathrm{i} \omega t}\right\} \mathfrak{R}\left\{q \mathrm{e}^{\mathrm{i} \omega t}\right\} d t=\frac{1}{2} \Re\left\{p_{0}^{*} q\right\}$

where $p_{0}^{*}$ indicates the complex conjugate of $p_{0}$ and $T=$ $2 \pi / \omega$ is the wave period. Here, we have assumed that the behavior of the air turbine is such that there is a linear relationship between the flux and the pressure in each chamber,

$q=\sum_{j=7}^{6+M_{p}} q_{j}, \quad q_{j}=B_{j 0} p_{j 0}$

where $p_{j 0}$ indicates the pressure, and $B_{j 0}$ the applied damping coefficient, on the chamber surface $S_{j}$ associated with mode $j$. The effects of air compressibility are also neglected with this assumption. In this case the total mean power extracted by each chamber over a cycle is given by

$W_{j}=\frac{1}{2} B_{j 0} p_{j 0}^{*} p_{j 0}$

The sum of $W_{j}$ over all chambers gives the total power extracted, and will normally be expressed as a capture width ratio by scaling it with the maximum available power passing through a section of the free surface of length $L_{c}$ along a wave crest,

$W_{\max }=\frac{1}{2} \rho g A^{2} c_{g} L_{c}$

where $L_{c}$ is usually taken to be either the wavelength $\lambda$ or the largest dimension of the body.

Combining (11) and (13) with the decompositions of (5) and (2), we can write a flux-pressure balance equation for each mean pressure mode $j$ in the form

$$
-B_{j 0} \rho g \xi_{j}-q_{j R}=q_{j D}
$$

The diffraction flux $q_{j D}$ is defined by (11), and using (4) we can write this as

$q_{j D}=\int_{S_{j}} \partial_{z} \phi_{D} d S=\frac{\omega^{2}}{g} \int_{S_{j}} \phi_{D} \mathrm{~d} S=\frac{\mathrm{i} \omega}{\rho g} X_{j}$ with

$X_{j}=-\mathrm{i} \omega \rho \int_{S_{b}} \phi_{D} n_{j} \mathrm{~d} S$

the standard WAMIT definition of the exciting force coefficient. Similarly for the radiation flux $q_{j R}$, we combine Eqs. (11), (10) and (5) to write

$$
\begin{aligned}
q_{j R} & =\int_{S_{j}} \partial_{z} \phi_{R} \mathrm{~d} S=\mathrm{i} \omega \sum_{k=1}^{6+M_{p}} \xi_{k} \int_{S_{j}} \partial_{z} \phi_{k} n_{j} \mathrm{~d} S \\
& =\mathrm{i} \omega \sum_{k=1}^{6+M_{p}} \xi_{k} \int_{S_{j}}\left(\frac{\omega^{2}}{g} \phi_{k}+n_{k}\right) n_{j} \mathrm{~d} S \\
& =\mathrm{i} \omega \sum_{k=1}^{6+M_{p}} \xi_{k}\left[\frac{\omega^{2}}{\rho g}\left(A_{j k}-\frac{\mathrm{i}}{\omega} B_{j k}\right)-c_{j k}\right]
\end{aligned}
$$

where

$c_{j k}=-\int_{S_{j}} n_{k} n_{j} \mathrm{~d} S$

and we have used the WAMIT definition of the added mass, and damping coefficients

$A_{j k}-\frac{\mathrm{i}}{\omega} B_{j k}=\rho \int_{S_{b}} \phi_{k} n_{j} \mathrm{~d} S$.

Note that due to the extended definition of the normal vectors, $c_{j k}=0$ when $j>6$, except along the diagonal where $c_{j j}=$ $-S_{j 0}$ is just the area of internal free surface $S_{j}$. Inserting (19) and (17) into (16) and dividing through by $\mathrm{i} \omega$ gives

$$
\begin{aligned}
& -\frac{\rho g B_{j 0}}{\mathrm{i} \omega} \xi_{j}+\sum_{k=1}^{6+M_{p}}\left[-\frac{\omega^{2}}{\rho g}\left(A_{j k}-\frac{\mathrm{i}}{\omega} B_{j k}\right)+c_{j k}\right] \xi_{k} \\
& =\frac{X_{j}}{\rho g} .
\end{aligned}
$$

This equation is in the same form as the standard rigidbody mode equations of motion, but with an applied external damping coefficient which is also a function of $\omega$. We can thus write the complete system of equations as

$$
\begin{aligned}
& \sum_{k=1}^{6+M_{p}}\left[-\bar{\omega}^{2}\left(\bar{M}_{j k}+\bar{A}_{j k}-\mathrm{i} \bar{B}_{j k}\right)-\frac{1}{\mathrm{i} \bar{\omega}} \bar{B}_{j k}^{0}+\bar{c}_{j k}\right] \bar{\xi}_{k} \\
& \quad=\bar{X}_{j}, \quad j=1,2, \ldots, 6+M_{p}
\end{aligned}
$$

where the matrix $\bar{M}_{j k}$ is the standard linearized body inertia matrix and the WAMIT non-dimensionalization has been 
applied in terms of a length-scale $L$, the gravitational constant $g$, the water density $\rho$ and the incident wave elevation A. Explicitly,

$$
\begin{aligned}
& \bar{A}_{j k}=\frac{A_{j k}}{\rho L^{m}}, \quad \bar{B}_{j k}=\frac{B_{j k}}{\rho \omega L^{m}} \quad \bar{X}_{j}=\frac{X_{j}}{\rho g L^{n}} ; \\
& \bar{\xi}_{j}=\frac{\xi_{j}}{A}, \quad \bar{B}_{j j}^{0}=\frac{\rho \sqrt{g L}}{L^{2}} B_{j 0}, \quad \bar{c}_{j j}=-\frac{S_{j 0}}{L^{2}}, \quad j>6 ;
\end{aligned}
$$

and $\bar{\omega}=\omega \sqrt{L / g}$. The pressure surface mode response $\xi_{j}$, $j>6$ is a pressure head measured in meters, i.e. it is a translational mode like surge, sway and yaw; as opposed to the rotational modes roll, pitch and yaw. Thus the exponents $m$ and $n$ here are as follows: $m=3$ for both $j, k=1,2,3,7, \ldots, 6+$ $M_{p}$ (translational-translational mode combinations); $m=5$ for both $j, k=4,5,6$ (rotational-rotational combinations); and $m=4$ for translational-rotational combinations; $n=2$ for translational modes and $n=3$ for rotational modes. We note that since the excess pressure developed in each OWC chamber must be produced by the floating structure itself, there will in general be hydrostatic coupling between the rigid-body modes and the pressure modes which must be included in the matrix $\bar{c}_{j k}$.

Having solved for the body response in the frequency domain, the capture width ratio with respect to a lengthalong-the-crest of $L_{c}$ can be written in terms of nondimensional quantities as

$\bar{W}=\frac{W}{W_{\max }}=\sum_{j=7}^{6+M_{p}} \frac{\bar{B}_{j 0} \bar{\xi}_{j}^{*} \bar{\xi}_{j}}{\bar{c}_{g}} \frac{L}{L_{c}}$

where $\bar{c}_{g}=c_{g} / \sqrt{g L}$.

If we consider a fixed, single degree of freedom OWC chamber, then the solution for the chamber response is explicit and simply given by

$\bar{\xi}_{7}=\frac{\bar{X}_{7}}{\left[-\bar{\omega}^{2} \bar{A}_{77}+\bar{c}_{77}+\mathrm{i}\left(\bar{\omega}^{2} \bar{B}_{77}+\frac{\bar{B}_{0}}{\bar{\omega}}\right)\right]}$.

The optimum PTO damping $\bar{B}_{0}$ in this case can be derived in the usual way by inserting (26) into (14) and setting the derivative with respect to $B_{0}$ to zero to get

$$
\left(\bar{B}_{0}\right)_{\mathrm{opt}}=\bar{\omega} \sqrt{\bar{c}_{77}^{2}-2 \bar{A}_{77} \bar{c}_{77} \bar{\omega}^{2}+\left(\bar{A}_{77}^{2}+\bar{B}_{77}^{2}\right) \bar{\omega}^{4}} .
$$

We note that at the undamped natural frequency of the chamber

$$
\bar{\omega}_{0}=\sqrt{\frac{\bar{c}_{77}}{\bar{A}_{77}}}
$$

i.e. when it is in resonance, the optimal damping becomes

$$
\left(\bar{B}_{0}\right)_{\mathrm{opt}}=\bar{\omega}^{3} \bar{B}_{77}
$$

which is analagous to the optimum condition for a single degree of freedom mechanical WEC which is tuned to resonance.

\subsection{Estimating the linearized PTO damping coefficient $B_{0}$}

The choice of the air turbine used to extract power from the chamber is an important aspect of the design of OWC devices, and an active area of ongoing research. The Wells-type turbine has traditionally been the most widely used, though impulse turbines are also available and the recently invented bi-radial turbine, see e.g. Falcão et al. (2013), appears to provide another promising alternative. The Wells turbine produces a linear pressure/flux relation which is convenient from a numerical modeling point of view, and it can be modeled in the laboratory by means of a suitably designed porous membrane covered orifice. The impulse turbine produces a quadratic relation between pressure and flux and is simple to model in the lab using an open orifice with an area of around $1 \%$ of the total interior free surface area. We note however, that air compressibility effects are generally important for full-scale devices which requires special care both numerically and at model scale. These issues are discussed at length in a recent publication by Falcão and Henriques (2014). Here, we have chosen to adopt a simple open orifice model.

To numerically model the orifice in the frequency domain requires the development of an equivalent linear damping coefficient. Ignoring compressibility effects which are insignificant at this scale, the pressure drop across the orifice is proportional to the square of the flow velocity (or flux) through the orifice

$p(t)=\frac{1}{2} \rho_{a}\left(\frac{1}{C_{d} a}\right)^{2} Q(t)^{2} \operatorname{sign}(Q)=\frac{1}{B_{1}} Q(t)^{2} \operatorname{sign}(Q)$

where $\rho_{a}=1.225 \mathrm{~kg} / \mathrm{m}^{3}$ is the air density, $a$ is the area of the orifice, and $C_{d}$ is an effective area coefficient which has been determined experimentally to be $C_{d}=0.64$ for the chamber and orifice tested here. Figure 2 plots the experimental results together with (30) and we can see that the relation gives a very good fit to the data. This relation can be used directly in the time domain, but to do the analysis in the frequency domain requires estimating an equivalent linear damping coefficient. To do this, we assume an oscillatory flux in the chamber

$Q(t)=|q| \sin (\omega t)$ 


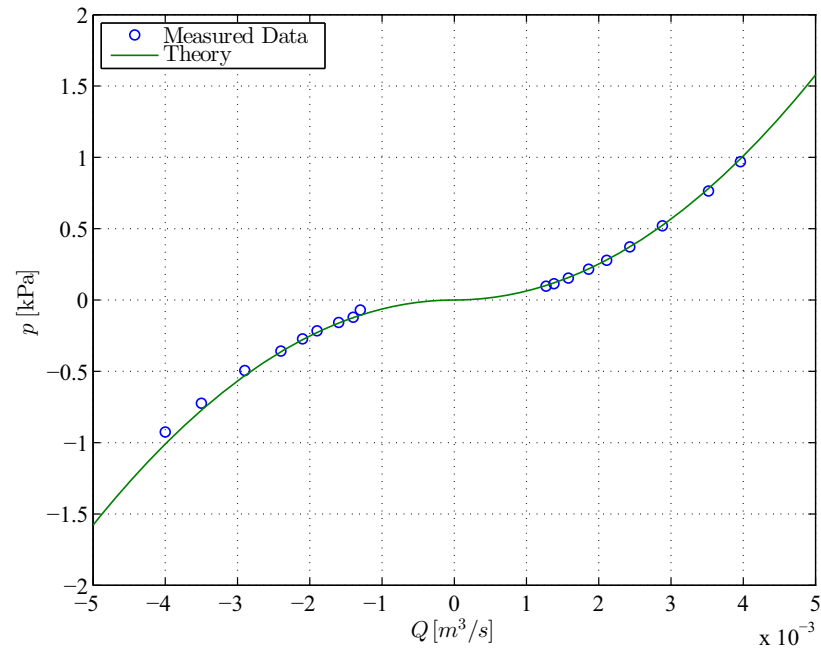

Fig. 2 Measured pressure drop across the chamber orifice vs volume flux through the chamber

and look for a $B_{0}$ which gives the same mean power loss over one cycle of oscillation. The mean power loss is given by

$W=\frac{1}{T} \int_{0}^{T} p(t) Q(t) \mathrm{d} t$.

Inserting first (30) and then (13) into (32), using (31), and finally equating the two results gives the relation

$B_{0}=\frac{3 \pi}{8|q|} B_{1}$

According to our linear model, the magnitude of the flux in the chamber is given by

$|q|=B_{0}\left|p_{0}\right|=\rho g\left|\xi_{7}\right| B_{0}$

which leads to

$B_{0}=\sqrt{\frac{3 \pi}{8 \rho g\left|\xi_{7}\right|} B_{1}}$

as the equivalent linearized damping coefficient. Figure 3 plots the result of (35) for the double chamber shown in Fig. 7, where we have solved iteratively for the chamber response shown in Fig. 4 at three values of wave steepness $H / \lambda=0.025,0.04$ and 0.06 . Also shown for reference is the optimum value from (27).

\section{The response of one fixed, double-chamber section}

We consider first the response of one fixed double-chamber section.

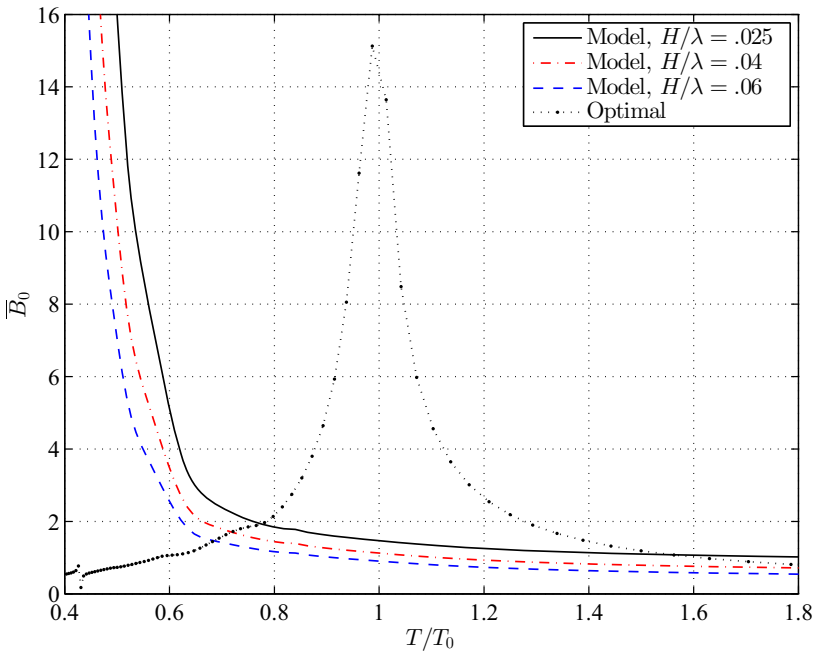

Fig. 3 Equivalent linearized damping coefficients from (35) corresponding to the response shown in Fig. 4 for one fixed double-chamber. The optimum value of (27) is also shown

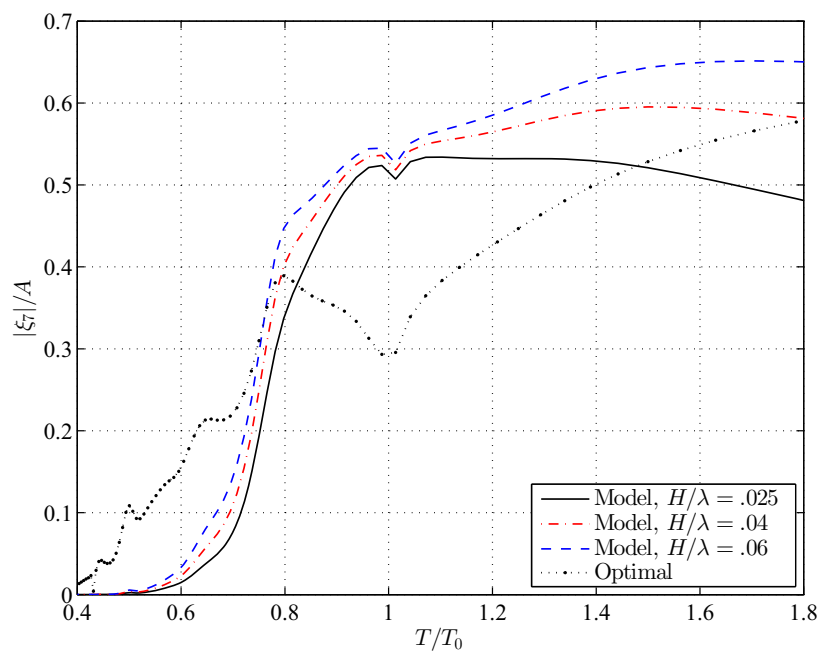

Fig. 4 Single chamber pressure response amplitude using the linearized PTO damping coefficient values shown in Fig. 3

\subsection{Experimental measurements}

We provide here a brief description of the experimental set up and analysis procedure, while more details can be found in Ducasse (2014). The physical model used in the experiments is shown in Fig. 5 and a schematic of the flume is shown in in Fig. 6. The full-scale internal chamber dimensions are $6 \mathrm{~m}$ by $5 \mathrm{~m}$ by $7.5 \mathrm{~m}$ in the $x, y$ and $z$-directions, respectively, and the model is at a scale of 1:50. These chamber dimensions give an undamped natural period of $5.9 \mathrm{~s}$ which is tuned to be close to a typical value for the conditions in the Danish North Sea near the northwest coast of Jylland. The flume used here measures $25 \mathrm{~m}$ by $0.6 \mathrm{~m}$, and the water depth was set to $0.65 \mathrm{~m}$. The width of the model is $0.1 \mathrm{~m}$, so there are only six chamber widths across the flume, and we can expect some 
wall effects to be present. The first transverse sloshing mode in the tank is at $T / T_{0}=0.74$ and an enhanced response near this point can be seen in the results shown below.

For these measurements, one tank wall was used as a symmetry plane and tests were made at a series of monochromatic conditions using three values of wave steepness corresponding to $H / \lambda=0.025,0.04$ and 0.06. A Smartec SPD102DAhyb analog pressure sensor attached to the chamber lid was used to measure the pressure inside the chamber. Two resistance wave probes were inserted into the chamber to measure the internal free surface elevation with the lid both on and off the chamber. These two measurements gave nearly

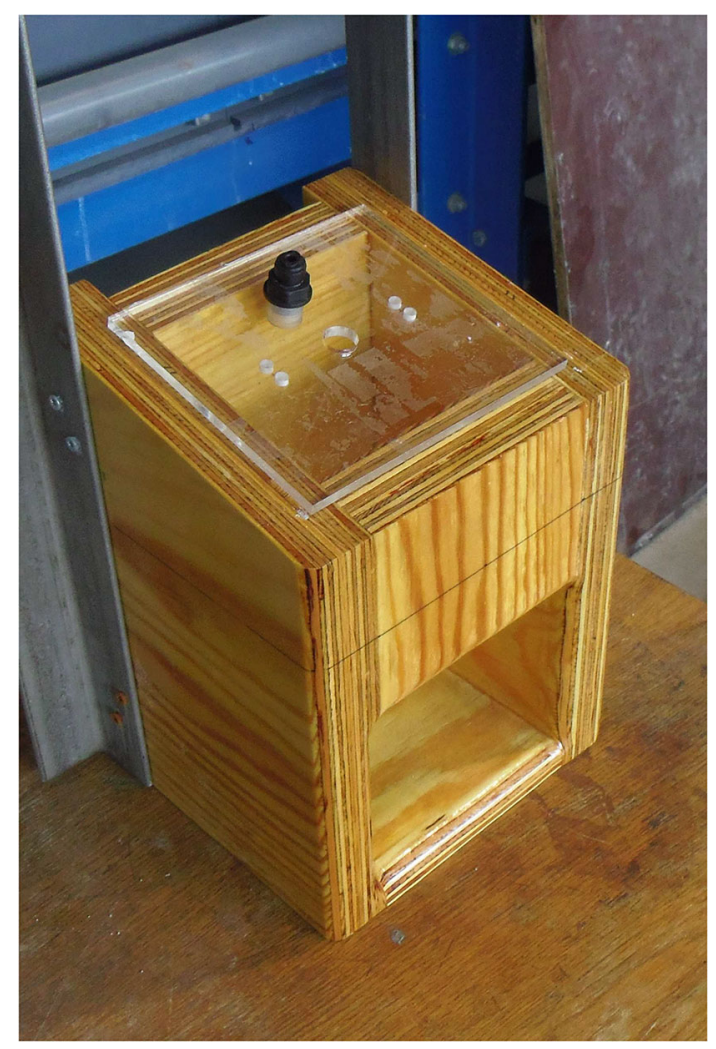

Fig. 5 The 1:50 scale model of one double-chamber section used in the DTU experiments. The tank wall was used as a symmetry plane, so only half of the section appears in the model. The water line is indicated by the black line identical results for all the closed chamber cases considered here. Visual inspection also confirmed that there was no obvious internal sloshing mode activity. We can, thus, expect that the inclusion of only one piston mode for each chamber is justified in this case. With the lid off the chamber, however, the violent motions near resonance did lead to sloshing mode activity and wave breaking.

To verify the repeatability of the wave conditions and establish the undisturbed wave amplitudes, all of the test conditions were first run multiple times without the chamber in place, and the wave elevations were recorded at the four positions along the tank indicated in Fig. 6. The same cases were then run again with the chamber in place and the following procedure was adopted for processing the data. The signals were first low-pass filtered to remove noise at frequencies higher than the sixth wave harmonic. To define a pressure or wave elevation amplitude, and to estimate the mean absorbed power, we considered a five wave period window of the total measured time series, chosen during the interval between the arrival of the wave front at the chamber and the time when any possible reflections from the ends of the tank could arrive back at the chamber. The amplitude used for the comparisons below is taken to be the first-harmonic amplitude of a least squares fit to each record using the first five harmonics.

Since we have measured both the pressure and the internal surface elevation in the chamber, the absorbed power can be computed from (32) in three ways: via the measured pressure and the time derivative of the measured internal chamber elevation; via the pressure alone and (30); or via the time derivative of the elevation alone and (30). These three methods give only small differences in the estimated average absorbed power. The measured absorbed power is finally multiplied by two to account for the image chamber.

\subsection{Calculations and results}

The WAMIT geometry used for the calculations is shown in Fig. 7. We first consider the free undamped internal surface elevation amplitude response of the chamber, which is shown in Fig. 8. This figure compares the measured data at the three values of incident wave steepness with the WAMIT
Fig. 6 Schematic of the experimental set up in the DTU flume, showing the locations of the chamber and the wave probes relative to the wavemaker and the beach. The tank is $0.6 \mathrm{~m}$ wide and the water depth is $0.65 \mathrm{~m}$

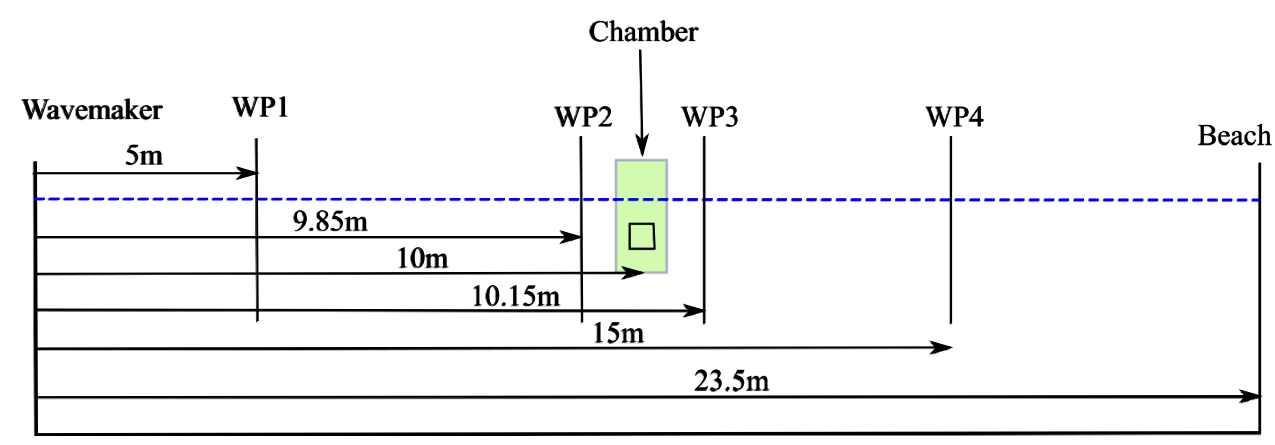




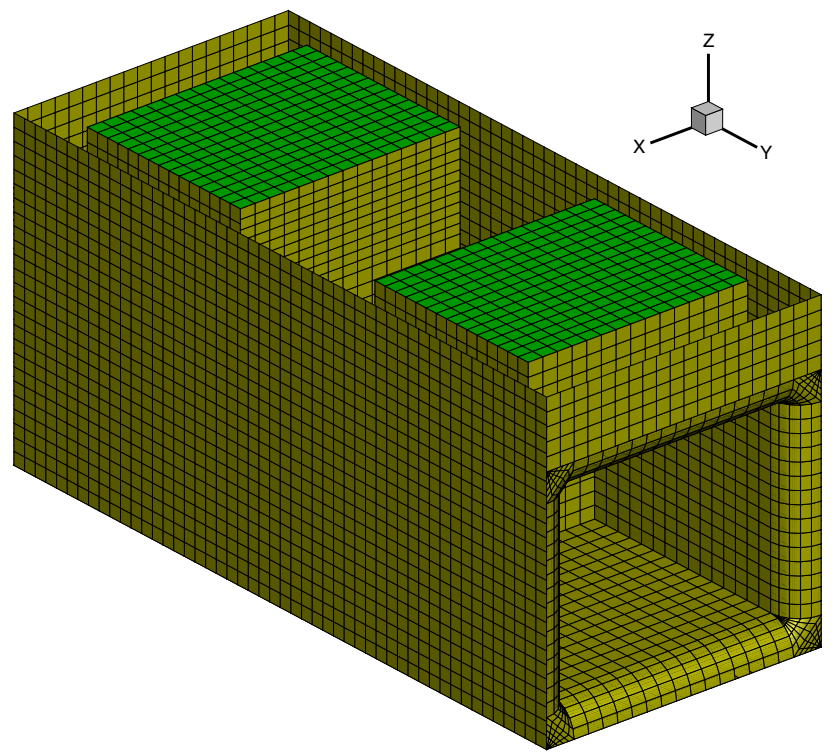

Fig. 7 The submerged geometry of the double-chamber section used for the WAMIT calculations. The green (bright) grid shows the internal pressure surface $S_{i}$, while the olive (dull) grid shows the solid body surface $S_{0}$. Geometry created using the MultiSurf software from AeroHydro (2014) (color figure online)

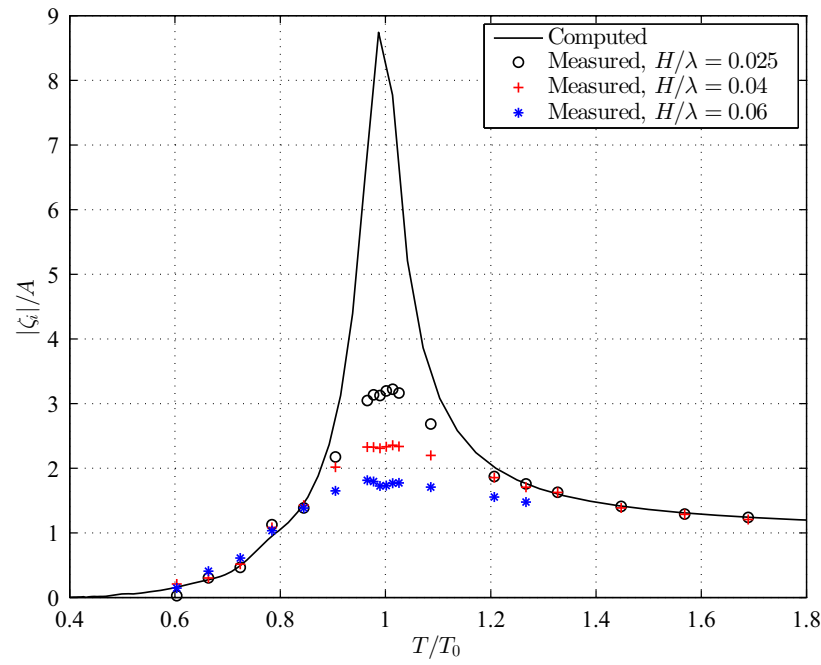

Fig. 8 Free undamped internal surface elevation amplitude response of the chamber plotted vs wave period

calculations. Near resonance, the internal chamber motions become large enough to splash over the top of the chamber and dip below the submerged entrance, leading to strongly nonlinear effects which are, of course, not captured by the calculations. Away from resonance, however, the agreement can be seen to be excellent.

The response amplitude for the internal pressure in the chamber with the lid attached is shown in Fig. 9. The calculations here are made using the values of equivalent linearized PTO damping coefficient shown in Fig. 3. Here, we can see that the pressures are somewhat over predicted near reso-

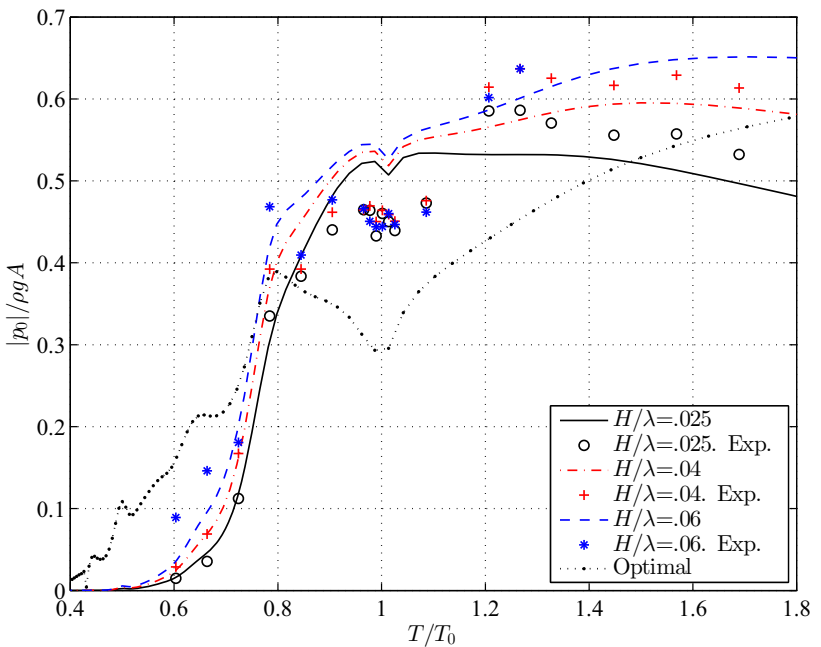

Fig. 9 The pressure amplitudes inside the chamber normalized by the incident wave amplitude $A$

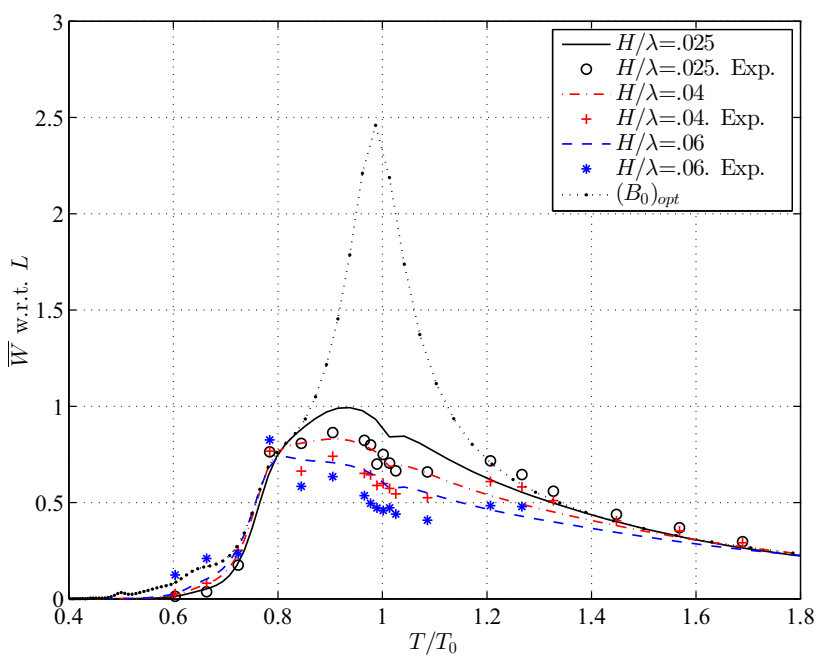

Fig. 10 The capture width ratio based on the length of the structure, $L=7.5 \mathrm{~m}$, using the PTO damping coefficients shown in Fig. 3

nance, and slightly under predicted in longer waves, but the general behavior and the trends with increasing wave steepness are well predicted. The total absorbed power is shown in Fig. 10 in the form of the capture width ratio based on the total length of the chamber in the $x$-direction, $L=7.5 \mathrm{~m}$. The comparison here can be seen to follow the internal pressure closely, as would be expected. The optimum absorption predicted by using (27) as the damping coefficient is also shown for reference, and we can see that the theory predicts significant improvement in the near vicinity of the resonant period. In all of these calculations, the finite tank width has been partially accounted for by including one tank wall in the WAMIT analysis. More images of the chamber could also be added to more fully account for the second wall, but this is left for future work. 


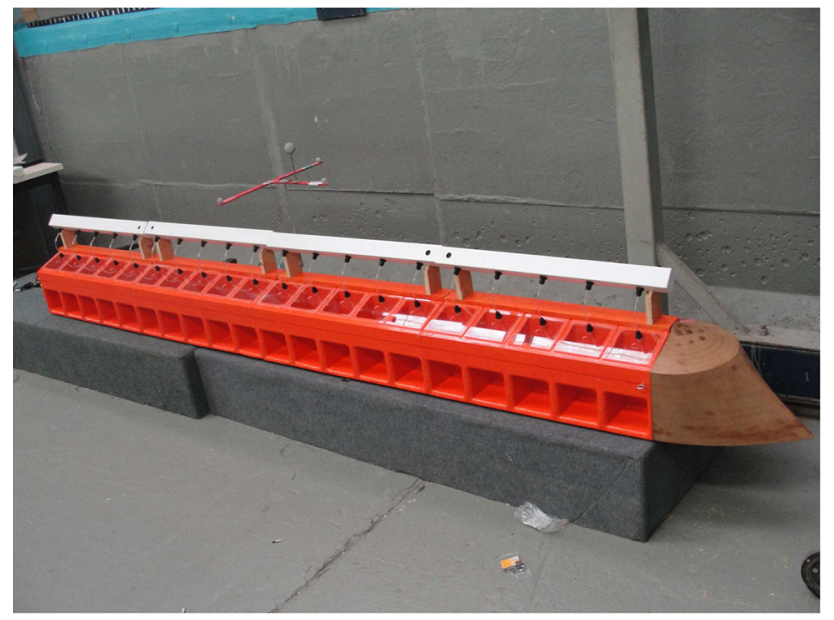

Fig. 11 Photo of the scale model ready for testing. A removable bow section is fitted in this picture, but results are presented here only without the bow

\section{The response of the full model}

We now consider the full 40 chamber model.

\subsection{Experiments}

The experiments on the full model were carried out at the Hydraulic and Maritime Research Center (HMRC) at University College Cork, Ireland in 2013. We describe the experiment briefly here while more details can be found in Nielsen (2013) and Pors and Simonsen (2013). A picture of the 1:50 scale model is shown in Fig. 11 and a schematic of the experimental set up is shown in Fig. 12. The basin measures $25 \mathrm{~m}$ by $18.5 \mathrm{~m}$ with a water depth of $1 \mathrm{~m}$. It has 40 hinged-flap, dry-backed, wave paddles for wave generation, and a $5 \mathrm{~m}$ long Enkamat beach for wave absorption at the far end. The model used in these experiments measures $3 \mathrm{~m}$ long by $0.345 \mathrm{~m}$ wide, with a draft of $0.165 \mathrm{~m}$, thus we do not expect the side walls to have any significant effect on the measurements in this case. A cluster of five wave probes was placed approximately $2 \mathrm{~m}$ in front of the centerline of the model and a horizontal line of nine probes at $1 \mathrm{~m}$ intervals was placed $2.25 \mathrm{~m}$ behind the model (see Fig. 12). The same basic experimental and analysis procedure was followed for these tests as the one described above for the single chamber tests. In this case, the model was tested in both regular and irregular waves and in both a fixed and a moored condition. The pressure in all 20 chambers along one side of the model was measured using pressure sensors and the rigidbody motions were measured using an optical system. The forces in the mooring system, which is indicated by the solid lines in Fig. 12, were also recorded. Tests were done both with and without the removable bow section which is shown in Fig. 11.

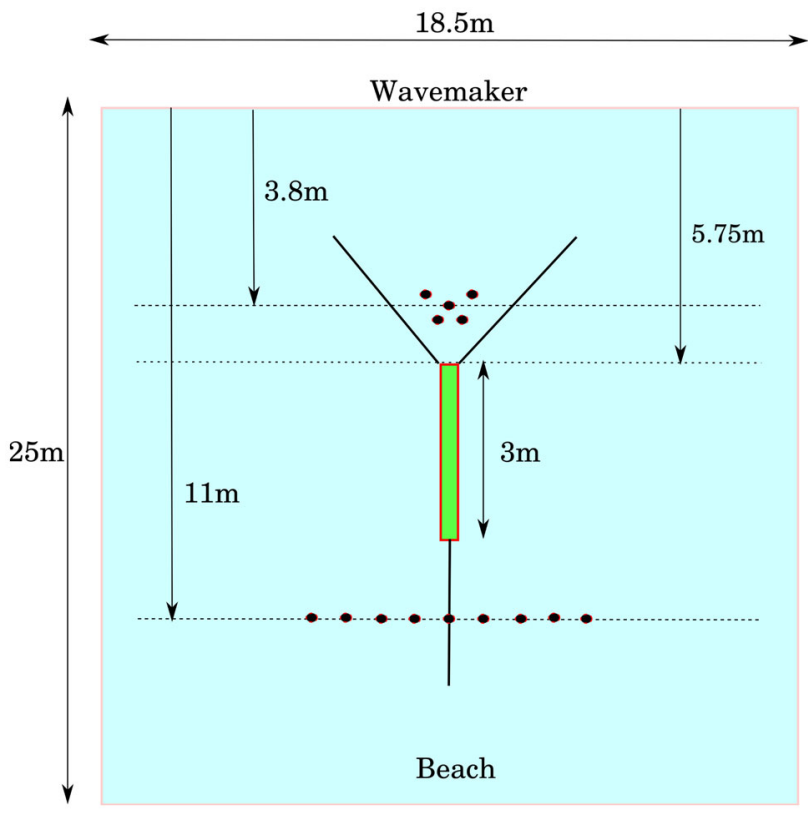

Fig. 12 Schematic of the experimental set up at HMRC. The solid lines indicate the mooring lines and the points indicate the wave gauges. The water depth is $1 \mathrm{~m}$ and the motions where measured by an optical system

In this paper, we will focus on the regular wave tests, without the bow section, in the fixed and the freely floating (slack-moored) conditions. As in the single chamber tests, the regular waves were tested at wave steepness values of $H / \lambda=0.025,0.04$ and 0.06 . The internal chamber surface elevations were not measured in these tests, so the absorbed power has been computed from the pressure measurements, together with the pressure-flux relation in (30).

\subsection{Fixed model calculations and results}

Figure 13 shows the high-order patch boundaries of the WAMIT geometry used for the calculations. This model was also created using the MultiSurf software. For the numerical solution in this case there are 20 degrees of freedom, one for each pair of chambers. To estimate the equivalent linearized damping coefficients for each chamber, the procedure described above is applied to iteratively find a set of coefficients and responses that satisfy (35). However, here an additional constraint has been added to limit the internal chamber free surface motion amplitude to $2.5 \mathrm{~m}$ (at full scale), i.e. the distance from the mean free surface to the top of the submerged chamber opening. From the assumed relation between pressure and flux in the chamber (13), this condition can be written

$$
\bar{\zeta}_{j}=\frac{-\bar{B}_{j 0}}{\mathrm{i} \omega\left|\bar{c}_{j j}\right|} \bar{\xi}_{j} \rightarrow\left|\bar{\xi}_{j}\right| \leq \frac{2.5}{A} \frac{\bar{\omega}\left|\bar{c}_{j j}\right|}{\bar{B}_{j 0}}
$$




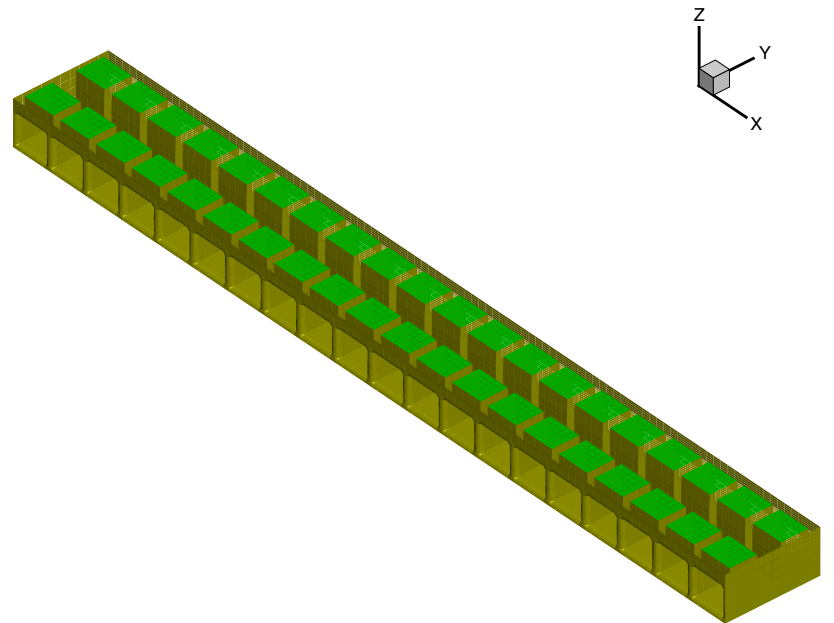

Fig. 13 The patch boundaries of the submerged geometry for the full 40 chamber numerical model. The green (bright) patches show the internal pressure surfaces of each chamber
The estimation of the optimal damping coefficients is now a non-linear optimization problem which has been solved using the Matlab constrained minimization routine fmincon based on the total absorbed power by all chambers with the above mentioned constraint on the maximum response amplitude.

Figure 14 shows the resultant damping coefficients and the pressure response in Chamber number 1, which is at the bow of the structure. Note that since the normalization length in this case is twenty times larger than that used for the single chamber case, the magnitude of $\bar{B}_{0}$ is much smaller here, even though $B_{0}$ itself is of same order of magnitude in both cases. The same data are shown in Figs. 15 and 16 for chamber number 10 just forward of the midships section, and chamber number 20 at the stern of the model. Experimental data for the fixed model is only available for the $H / \lambda=0.025$ case and this is also plotted. The agreement between theory and measurements is reasonably good, though the calculations tend to generally over-predict the pressures. The numerically optimized values are also plotted, and we can see that the behavior
Fig. 14 Bow chamber: the equivalent linearized damping coefficient (left) and the pressure response (right) for the fixed model

Fig. 15 Midships chamber: the equivalent linearized damping coefficient (left) and the pressure response (right) for the fixed model
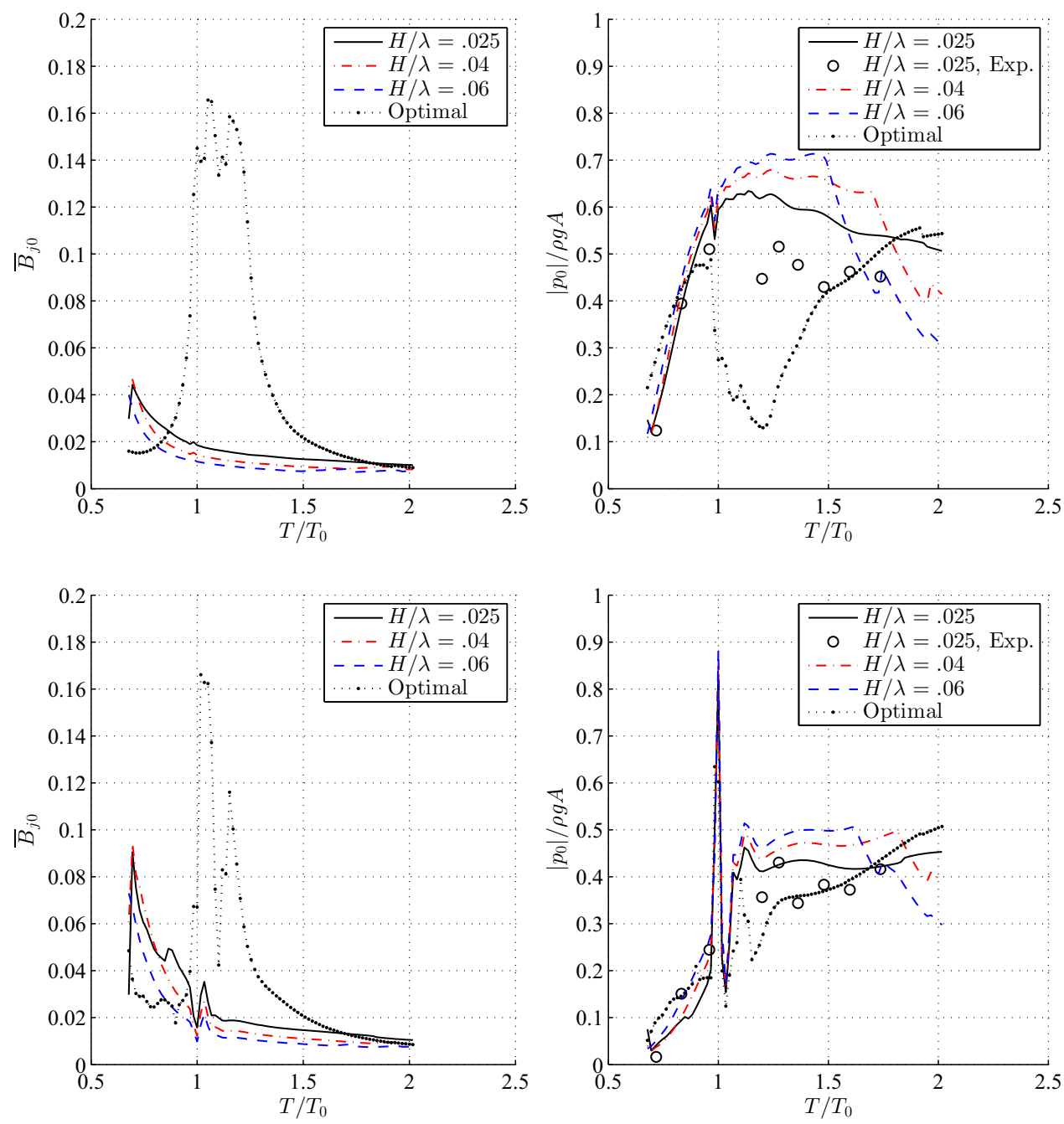
Fig. 16 Stern chamber: the equivalent linearized damping coefficient (left) and the pressure response (right) for the fixed model
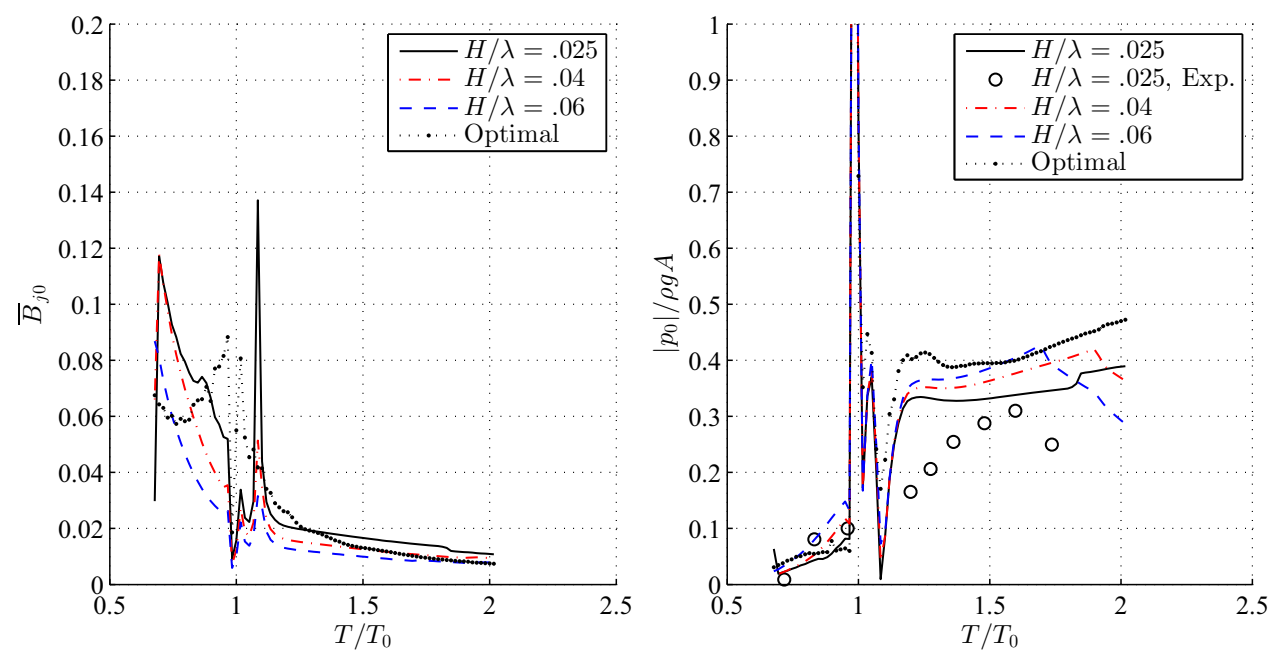

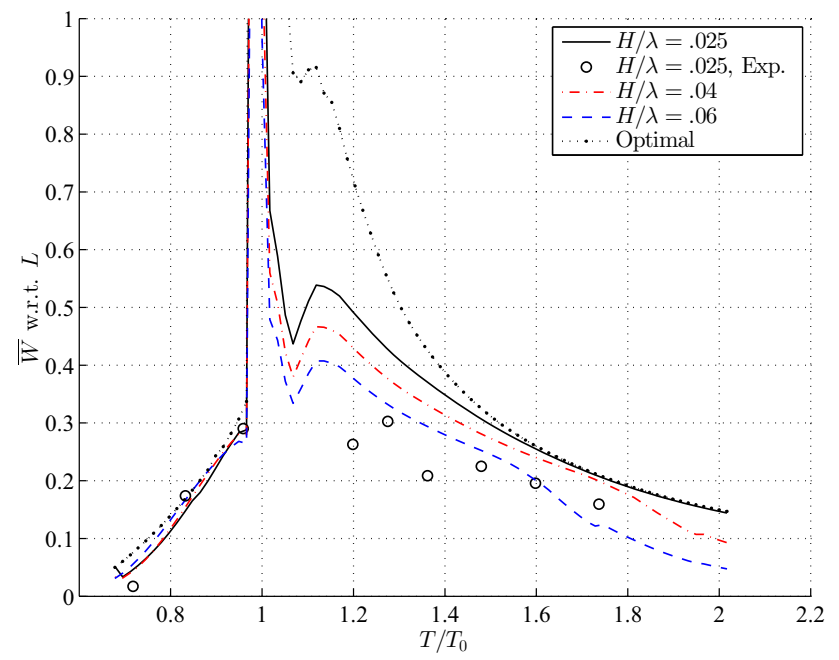

Fig. 17 The capture width ratio of the fixed 40 chamber model with respect to $L=150 \mathrm{~m}$

of the damping coefficients and the pressure responses are qualitatively similar to that of the single chamber, though the amplitudes tend to decrease as we move from bow to stern.

Figure 17 shows the absorbed power as a capture width ratio normalized by the total length of the device $L=150 \mathrm{~m}$. The general over-prediction of the chamber pressures leads, as would be expected, to a general over-prediction of the device absorption width for wave periods above the resonant period. As for the single chamber case, substantial improvement in absorption is predicted near the resonant period when an optimal damping coefficient is applied. Unfortunately there is a gap in the experimental data near the resonance period, but new experiments are in progress to fill in this region.

\subsection{Freely floating model calculations and results}

In the freely floating case, we add the six rigid-body modes to the 20 pressure-surface modes to get 26 degrees of free- dom. The rigid-body motions of the structure also induce flux in the chambers, so the pressure response $\xi_{j}$ is now the combined result of the rigid-body motions and the internal surface motion. The internal surface elevation $\zeta_{j}$, measured relative to the mean water line of chamber $j$, is thus given by

$\bar{q}_{j}=\left|\bar{c}_{j j}\right| \mathrm{i} \bar{\omega} \bar{\zeta}_{j}, \quad \bar{\zeta}_{j}=\bar{\zeta}_{j 0}-\bar{\xi}_{3}+\bar{x}_{j} \bar{\xi}_{5}+\bar{y}_{j} \bar{\xi}_{4}$

where $\bar{\zeta}_{j 0}=\zeta_{j 0} / A$ is the non-dimensional surface elevation amplitude relative to the still water level, and $\xi_{3}, \xi_{4}, \xi_{5}$ represent the heave, roll and pitch motion amplitudes respectively. If the non-dimensional $x$ and $y$-coordinates of the center of chamber $j$ are given by $\bar{x}_{j}, \bar{y}_{j}$, then the hydrostatic coupling between the pressure response and the heave, pitch and roll modes is given by

$\bar{c}_{3 k}=\bar{S}_{k 0} ; \quad \bar{c}_{4 k}=\bar{y}_{k} \bar{S}_{k 0} ; \quad \bar{c}_{5 k}=-\bar{x}_{k} \bar{S}_{k 0}$, for $6<k<=M_{p}$

This interaction is symmetric, so these coefficients are also reflected across the diagonal of the $\bar{c}_{j k}$ matrix. With these modifications, the solution proceeds as described above in the fixed body case.

The results for the damping coefficients and pressures in the bow, mid-section and stern chambers are shown in Figs. 18, 19 and 20. The experimental results for the measured pressures are also shown. The agreement between calculations and measurements is satisfactory with a general over-prediction near the resonant period and some underprediction in long waves. It is clear that the motions have a significant effect on the local pressures in the chambers compared to the fixed body case.

Figure 21 shows the absorbed power as a capture width ratio normalized by the total length of device $L=150 \mathrm{~m}$. The agreement here is perhaps slightly better than in the fixed model case. Interestingly, both calculations and exper- 
Fig. 18 Bow chamber: the equivalent linearized damping coefficient (left) and the pressure response (right) for the freely floating model

Fig. 19 Midships chamber: the equivalent linearized damping coefficient (left) and the pressure response (right) for the freely floating model

Fig. 20 Stern chamber: the equivalent linearized damping coefficient (left) and the pressure response (right) for the freely floating model
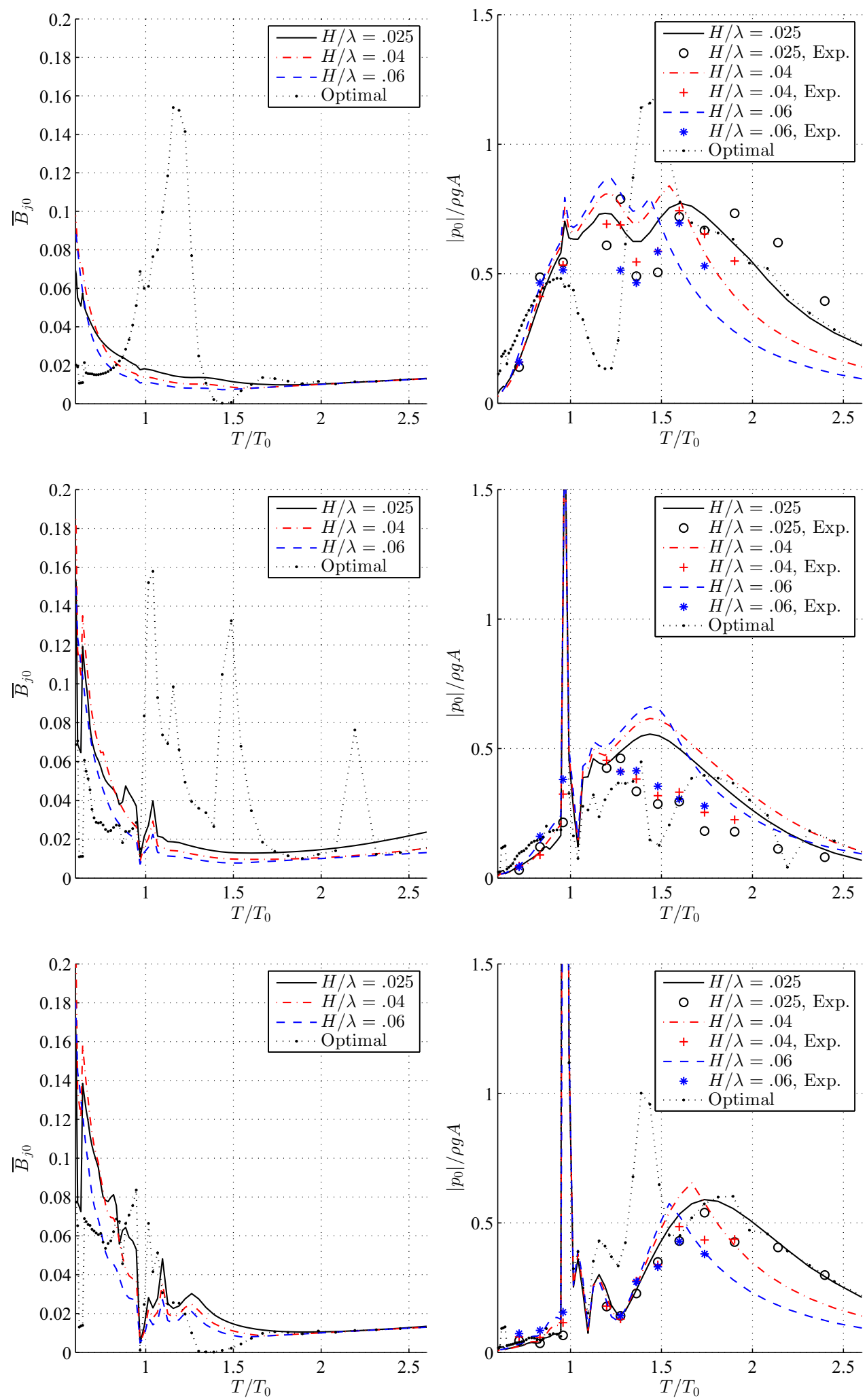
iments predict that the motions have a positive effect on the absorption near the resonant frequency, but a negative effect in long waves. The optimized damping coefficients predict a similar improvement in absorption to that which was found in the fixed model case. The left hand plot in Fig. 22 shows the same calculations normalized with respect to the incident wavelength $\lambda$ for reference. The right hand plot in Fig. 22 shows the predicted mean absorbed power in megawatts at full scale as a function of wave period for the three considered wave steepness values. This gives an indication of the real potential of such a device and also illustrates the large variability in absorbed power inherent to wave energy. As discussed above, a maximum of about $80 \%$ of this absorbed power will be converted into electrical power depending on the conditions and the actual air turbines used. It could also be attractive to consider using valves to

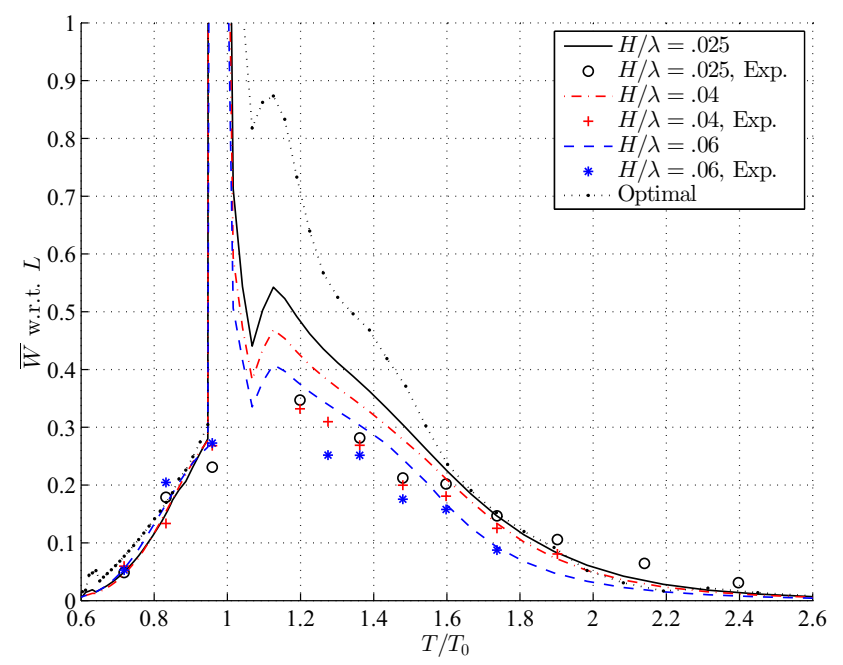

Fig. 21 The capture width ratio of the moored 40 chamber model with respect to $L=150 \mathrm{~m}$

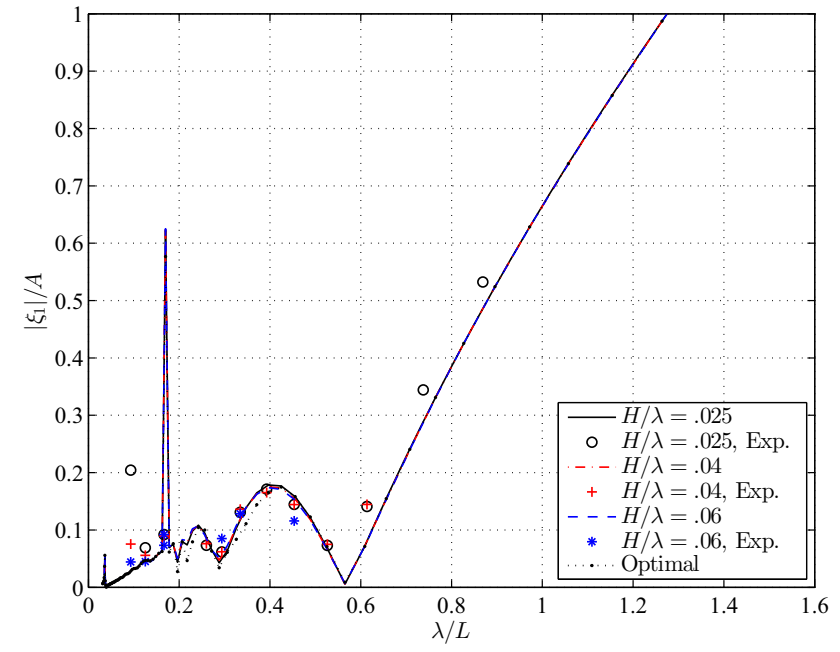

Fig. 23 The surge motion response of the full 40 chamber model

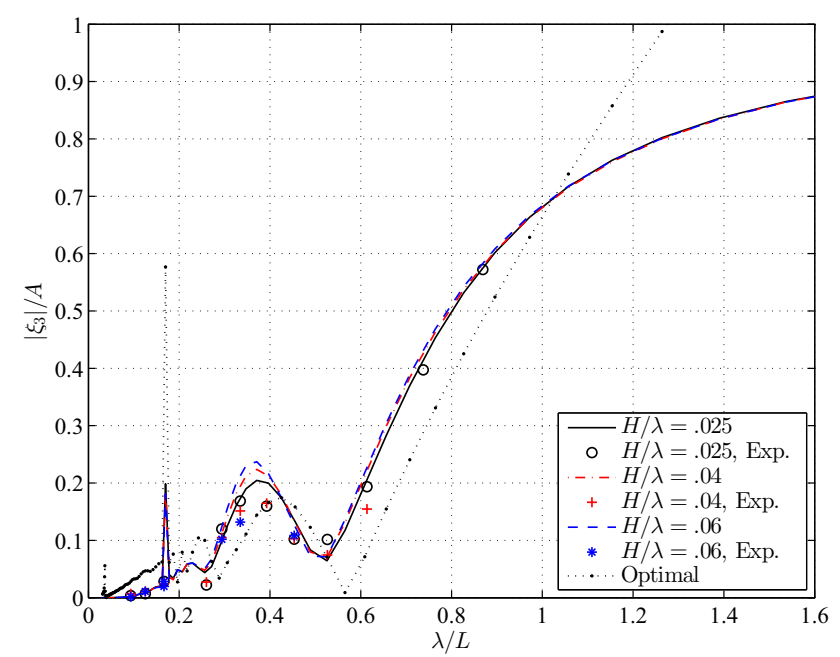

Fig. 24 The heave motion response of the full 40 chamber model
Fig. 22 The capture width ratio of the freely floating 40 chamber model with respect to the wavelength $\lambda$ (left), and the predicted full-scale power absorption in megawatts (right)
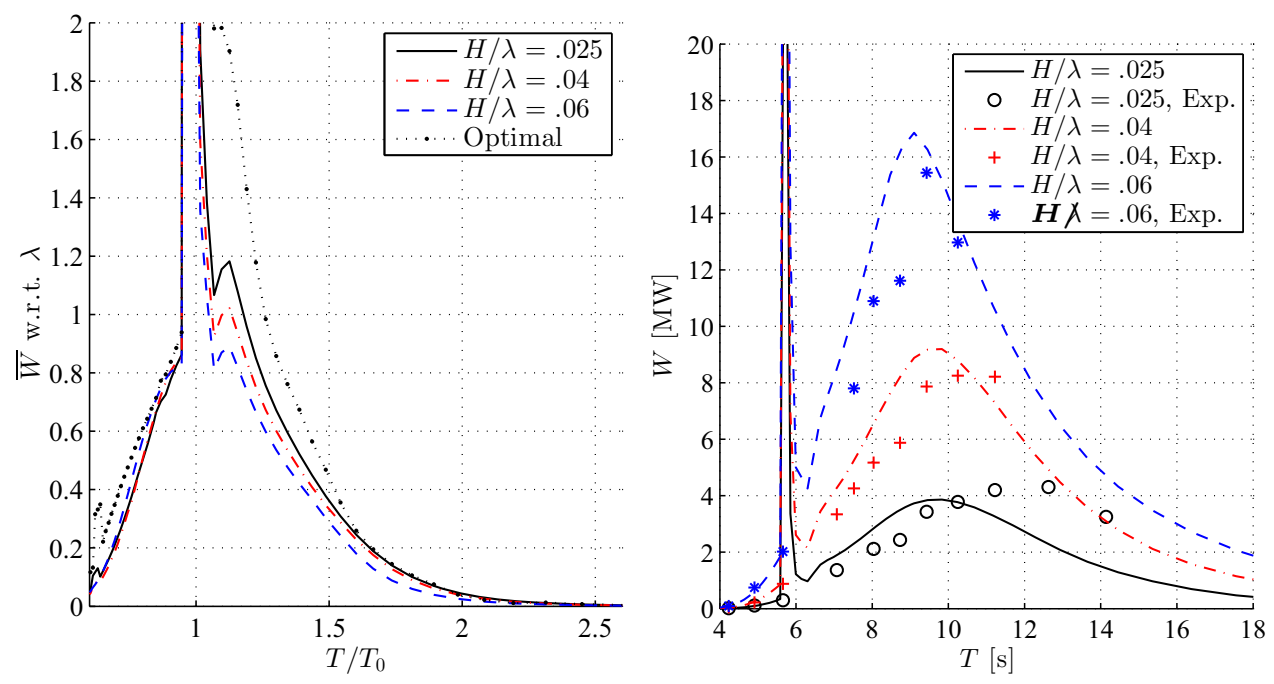


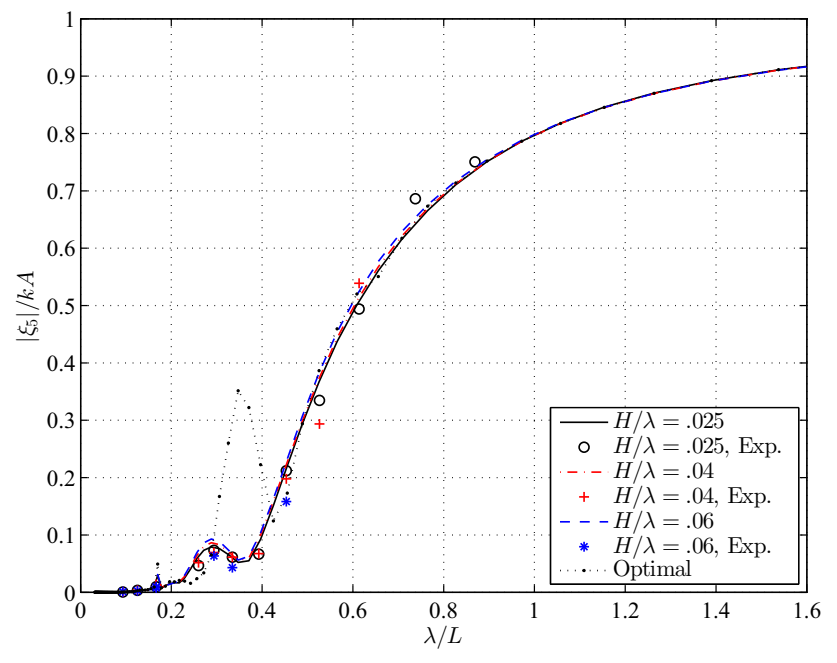

Fig. 25 The pitch motion response of the full 40 chamber model

connect all the chambers to a central channel to drive one unidirectional flow through a larger and more efficient turbine.

Figures 23,24 and 25 show the rigid-body motion response of the structure in the surge, heave and pitch modes, respectively. These results are normalized by the wave amplitude $A$ and the wave slope $k A$ for translational and rotational modes, respectively, and are plotted versus the relative incident wavelength. Here, we can see a good agreement between the calculations and the measurements, especially in the target wave period region where the rigidbody motions are small. The increased pressures in the chambers near resonance which are predicted by the optimized damping coefficients can be seen to introduce an enhanced pitch response there. We note that the mooring system introduces a very long natural period to the surge response of the structure, but this period is far beyond the wind-wave spectrum which we focus on here. We note also that the vertical center of gravity and the pitch radius of gyration of the ballasted model were used in the calculations.

\section{Conclusions}

In this paper, we have clarified several subtle details which are important to getting good performance out of standard frequency domain, linear potential flow theory for the analysis of floating structures which include one or more OWC degrees of freedom. This has been illustrated by considering both a fixed single degree of freedom OWC and an I-beam attenuator OWC device with 26 degrees of freedom. The agreement between calculations and measurements is generally found to be excellent, though the calculations tend to slightly over-predict the internal chamber pressures and therefore the absorbed power near resonance, but under- predict these quantities in long waves in the freely floating condition.

Future work will focus on weakly non-linear calculations in the time domain which will allow for a better model of the air turbine and inclusion of some viscous and air compressibility effects. Possible improvements to the design which are currently under investigation include: replacing the simple chamber with a U-OWC chamber; replacing the OWC with a simple mechanical oscillator; and introducing a valve system to rectify the air flow from all chambers to a central plenum and one large unidirectional turbine. Survivability tests, structural calculations and detailed mooring system design are also in progress to get an accurate estimate of the final cost of energy for such a system.

Acknowledgments The authors would like to thank Pablo Guillen for generating the 40 chamber geometry, and Frederik Pors Jakobsen and Morten Simonsen for building the models and carrying out the experimental measurements on the 40 chamber model. The HMRC experiments received support from MARINET, a European Community-Research Infrastructure Action under the FP7 "Capacities" Specific Programme.

\section{References}

AeroHydro (2014) Relational 3D modeling for marine and industrial design. http://aerohydro.com. Accessed 1 Jan 2014

Barstow S, Mørk G, Mollison D, Cruz J (2008) The wave energy resource. In: Cruz J (ed) Ocean wave energy, Current status and future perspectives. Springer, Berlin

Boccotti P (2007) Comparison besteen a U-OWC and a conventional OWC. Ocean Eng 34:799-805

Boccotti P, Filianoti P, Fiamma V, Arena F (2007) Caisson breakwaters embodying an OWC with a small opening. Part II: a small-scale field experiment. Ocean Eng 34:820-841

Boccotti P (2012) Design of breakwaters for conversion of wave energy into electrical energy. Ocean Eng 51:106-118

Boccotti P (2015) Wave mechanics and wave loads on marine structures. Butterworth-Heinemann, Amsterdam

CarbonTrust (2005) Oscillating water column wave energy converter evaluation report. http://www.carbontrust.com/media/173555/ owc-report.pdf. Accessed 1 Jan 2014

Cruz J (2008) Ocean wave energy, Current status and future perspectives. Springer, Berlin

Drew B, Plummer AR, Sahinkaya M (2009) A review of wave energy converter technology. Proc Inst Mech Eng Part A-J Power Energy 223:887-902

Ducasse D (2014) A theoretical and experimental study of an oscillating water column attenuator. Master's thesis, Technical University of Denmark, Lyngby

Evans DV (1982) Wave-power absoption by systems of oscillating surface pressure distributions. J Fluid Mech 114:481-499

Falcão AFO (2010) Wave energy utilization: a review of the technologies. Renew Sustain Energy Rev 14:899-918

Falcão AFO, Gato LMC, Nunes EPAS (2013) A novel self-rectifying air turbine for use in wave energy converters. Renew Energy 50:289 298

Falcão AFO, Henriques JCC (2014) Model-prototype similarity of oscillating-water-column wave energy converters. Int $\mathrm{J}$ Mar Energy 6:18-34 
Falnes J (2002) Ocean waves and oscillating systems. Cambridge University Press, Cambridge

Heath T (2012) A review of oscillating water columns. Phil Trans R Soc Lond A 370:235-245

IEA (2014) OES - ocean energy systems, an international energy agency technology initiative. http://www.ocean-energy-systems. org. Accessed 1 Jan 2014

Ishii S, Miyazaki T, Masuda Y, Kai G (1982) Reports and future plans for the kaimei project. In: Proc 2nd international symposium on wave energy utilization, Trondheim, Norway

Jacobsen P (2011) Mapping and assessment of the United States ocean wave energy resource. In: Technical report 1024637. Electric Power Research Institute, Palo Alto

Lee C-H, Newman JN, Nielsen FG (1996) Wave interactions with an oscillating water column. In: 6th intl. offshore and polar engineering conference, Los Angeles, USA

Lee C-H, Nielsen FG (1996) Analysis of oscillating water column device using a panel method. In: 11th intl wrkshp water waves and floating bodies, Hamburg, Germany. http://www.iwwwfb.org. Accessed 1 Jan 2014

López I, Andreu J, Ceballos S, Martinez de Alegria I, Kortabarria I (2013) Review of wave energy technologies and the necessary power-equipment. Renew Sustain Energy Rev 27:413-434

Masuda Y (1979) Experimental full scale result of wave power machine Kaimei in 1978. In: Proc. 1st inter symp on wave energy utilization, Gothenburg, Sweden

McCormick M (2007) Ocean wave energy conversion. Dover, Mineola
Moody GW (1980) The NEL oscillating water column: recent developments. In: First symposium on wave energy utilization, Oct 1979. Chalmers University of Technology, Gothenburg, pp 283-296

Newman JN, Lee C-H (2014) WAMIT; a radiation-diffraction panel program for wave-body interactions. http://www.wamit.com. Accessed 1 Jan 2014

Nielsen K (2013) Final report: KNSWING attenuator development phase I. Technical report, FP7-MARINET, marine renewables infrastructure network. http://www.fp7-marinet.eu/. Accessed 1 Jan 2014

OEE (2014) Ocean energy Europe, a trade association for Ocean renewables. http://www.oceanenergy-europe.eu/index.php/en/. Accessed 1 Jan 2014

Oyster (2014) Aquamarine power. http://aquamarinepower.com/. Accessed 1 Jan 2014

Pelamis (2014) Pelamis wave power. http://www.pelamiswave.com/. Accessed 1 Jan 2014

Pors F, Simonsen M (2013) A theoretical and experimental study of an oscillating water column attenuator. BS thesis, Technical University of Denmark, Lyngby

SIOcean (2012) Ocean energy: state of the art. Strategic initiative for Ocean energy. http://si-ocean.eu. Accessed 1 Jan 2014

WAVENERGY.IT (2014) WAVENERGY.IT S.r.l. http://wavenergy.it/. Accessed 1 Jan 2014

Wavestar (2014) Wavestar energy. http://wavestarenergy.com/. Accessed 1 Jan 2014 